Unfallchirurg 2010 $\cdot 113: 1058$

DOI 10.1007/s00113-010-1802-z

Online publiziert: 28. November 2010

(c) Springer-Verlag 2010

\section{H. Lill}

Klinik für Unfall- und Wiederherstellungschirurgie,

Diakoniekrankenhaus Friederikenstift gGmbH, Hannover

\title{
Was ist eine minimalinvasive Operationstechnik zur Versorgung proximaler Humerusfrakturen?
}

ten. Unklar bleibt, warum die Autoren nicht den anterolateralen Deltasplitzugang verwenden, der sich des vorderen Deltaseptums bedient, am vorderen Akromionrand beginnt und direkt über der Mitte des Humeruskopfs weiterläuft. Gerade beim Deltasplitzugang fängt der Hautschnitt häufig sehr klein an und wird dann während der Operation immer größer. Schmale Haken können dabei wie ein Messer wirken und die Deltamuskelfasern einschneiden. Eine vergleichende Studie [1] zwischen deltoideopektoralem und anterolateralem Deltasplitzugang konnte zeigen, dass 12 Monate postoperativ Vorteile des vorderen Zugangs bestehen.

In der Arbeit von Neubauer u. Wagner wird der N. axillaris unterfahren, um die Platte einzuschieben. Dies ist überflüssig und birgt eher die Gefahr der Verletzung des N. axillaris als dessen Schonung. Bleibt man direkt am Knochen mit der Platte und kommt man von vorne, kann der N. axillaris in der Regel nicht verletzt werden.

Was ist eine minimalinvasive Repositionstechnik? Eine in der Arbeit von Neubauer $\mathrm{u}$. Wagner dargestellte Repositionstechnik mit Kirschner-Drähten und zusätzlicher Schraubenfixation scheint mir auch in diesem Zusammenhang nicht als minimalinvasiv darstellbar zu sein. Das Problem der temporären Drähte besteht darin, dass diese häufig die Reposition bzw. korrekte Plattenposition behindern. Besser erscheinen die Reposition mit einem Elevatorium für die Kalotte, die ich in diesem Artikel vermisse, und die Fadencerclage zur Reposition und Zuggurtung der Tuberkula. Hiermit kann man den „Rahmen“ der Tuberkula schlie- ßen und dann die Kalotte in Abhängigkeit von der Fraktur vorher oder nachher reponieren.

Mittlerweile werden viele sog. minimalinvasive Operationstechniken am proximalen Humerus beschrieben, die aber nicht kritiklos als solche bezeichnet werden können. Minimalinvasiv am proximalen Humerus heißt: Anatomischer Zugang unter Schonung der Weichteilstrukturen, sorgfältige Reposition unter Respektierung der Biologie und eine stabile Osteosynthese.

\section{Korrespondenzadresse \\ Prof. Dr. H. Lill}

Klinik für Unfall- und Wiederherstellungschirurgie, Diakoniekrankenhaus Friederikenstift gGmbH

Postfach 2040

30020 Hannover

helmut.lill@ddh-gruppe.de

Interessenkonflikt. Der korrespondierende Autor gibt an, dass kein Interessenkonflikt besteht.

\section{Literatur}

1. Hepp P, Theopold J, Voigt C et al (2008) The surgical approach for locking plate ostheosynthesis of displaced proximal humeral fractures influences the functional outcome. J Shoulder Elbow Surg 17(1):21-28 